\title{
THE PATHOLOGICAL CHANGES IN RATS' NERVES SUBJECT TO MODERATE COMPRESSION
}

\author{
H. Weisl, Cardiff, Wales, and G. V. Osborne, Liverpool, England \\ From the Prince of Wales Orthopaedic Hospital, Rhydlafar, Cardiff, \\ and the Welsh National School of Medicine, Cardiff
}

In recent years there has been considerable interest in conditions such as carpal tunnel compression and ulnar neuritis which are believed to be due to gentle compression or constriction of peripheral nerves. Many of these lesions have been explored, and usually the nerve is found swollen proximal to the constriction and narrowed deep to it. Since the symptoms caused by the nerve compression are readily relieved by dividing the constricting agent, there is no opportunity for removing tissue for histological examination; consequently the pathological changes in the compressed nerves have not been widely studied. In this investigation, because of the lack of suitable human material, lesions resembling those seen in the nerve compression syndromes were induced in rats, and the histology and vascularity of these experimental lesions was examined.

\section{REVIEW OF PREVIOUS REPORTS}

Marie and Foix (1913) described about twenty cases of isolated atrophy of the thenar muscles, which they attributed to compression of the median nerve by the flexor retinaculum. From one patient with severe bilateral muscle wasting, presumably of long standing, they were able to obtain the median nerves at necropsy. They found the nerves swollen and indurated proximal to the retinaculum, the swelling consisting of fibrous tissue situated between and within the bundles of axons. Dustin (1917) recorded the pathological changes in compressed nerves which had been removed from war casualties. He found that the nerves were swollen not only proximal to the constriction but also distal to it. He ascribed this swelling to an accumulation of fluid secondary to the obstruction of neural lymphatics, which he assumed followed the nerves and drained directly into the cerebrospinal fluid; this accumulation of fluid was followed by intraneural fibrosis. He also found that the constricted nerves were traversed by newly formed arterioles.

This is all the human material that has been available for examination, and the lesions studied were much more severe than those generally seen in carpal tunnel syndrome and in related conditions.

The more recent investigations concern experimental lesions in warm-blooded animals. Weiss and his colleagues (Weiss 1943, 1944, Weiss and Davis 1943, Weiss and Hiscoe 1948), seeking a quick and simple technique of repairing cut nerves, tried to splice them with short lengths of tightly fitting arteries. They found that the nerves became swollen proximal and distal to the arterial sheaths. They attributed the proximal swelling to the obstruction of a hypothetical, proximo-distal flow of fluid within the axon, and they ascribed the distal swelling to degenerative changes following division of the nerve. They were uncertain whether this " axonal " fluid distended the axons or accumulated between them. Weiss (1943) also observed changes in the vascularity of the nerves but regarded these changes as transient. Denny-Brown and Brenner (1944) carried out experiments on cats in which pressure of known magnitude was applied to nerves by metal spring clips. Their findings were in some respects vitiated by the " intense inflammatory reaction" caused by the metal, but they did find that clips exerting a pressure of 2 to 7 grammes, while not interfering with conduction, caused swelling both 
proximal and distal to the constriction after two weeks. In the swollen parts the axons were packed less closely than normal, the increased space between them being occupied by " axonal " fluid, and the diameter of the axons was increased; in the constricted parts the axons were narrowed and packed more closely. Denny-Brown and Brenner did not subscribe to the hypothesis that the swelling of the nerve was due to a damming up of an axoplasmal flow.
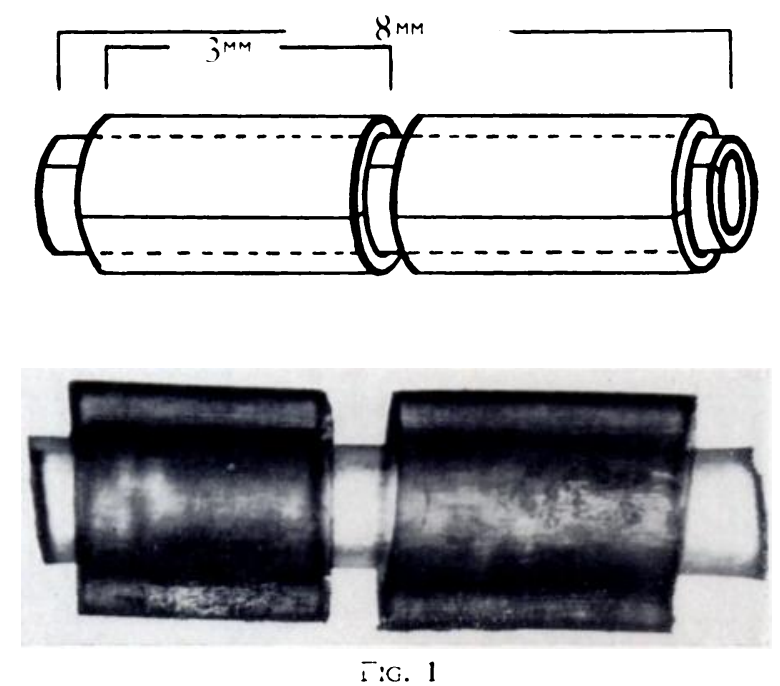

Diagram and photograph of the compression device. This consists of an inner piece of Number 2 Sterivac polythene tubing 8 millimetres long and two outer cuffs of Number 3 tubing 3 millimetres long. To allow this device to be placed on the nerve the tubes have been slit longitudinally.

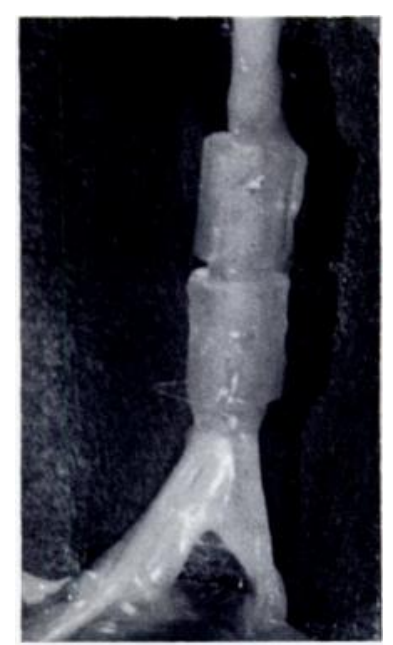

FIG. 2

The polythene compression device placed on the sciatic nerve (rat 150); proximal and distal swellings can be discerned.

On the basis of Causey's (1948) work it might be suggested that the swelling of the nerve was caused by mechanical factors. In his experiments rabbits' nerves were subjected to pressures of 50 to 670 millimetres of mercury until motor conduction in them failed; under these conditions the diameter of the axons was increased proximal and distal to the compression and Causey thought that this was caused by displacement of axonal material. However, these experimental conditions did not reproduce-nor were they intended to reproduce-the gentle constriction occurring in carpal tunnel syndrome and ulnar neuritis. It is equally doubtful whether the other workers were investigating the mild type of lesion seen in the nerve compression syndromes. In most of Weiss's experiments the nerve had been divided completely, and the conductivity of the constricted segment could not be studied. We repeated DennyBrown's and Brenner's experiments in a preliminary study, but found that the inflammatory changes were so intense that the histological changes these workers had reported could well be attributed to a reaction to the metal of the spring clips.

In the present research these objections have been overcome. A technique has been devised which allowed pressure to be applied to the nerve without damaging it severely, and the compression device was made of material which produced only the minimum of tissue reaction; this device was also of a size which allowed it to be implanted in the hind limb of a rat. The lesions produced by this device resembled macroscopically and electromyographically those seen in nerve compression in man. Microscopic examination of the swollen parts of the experimentally compressed nerves confirmed that the swelling of the nerve was largely caused by an accumulation of fluid between the axons, although the size of the axons was also increased. Further study proved that this fluid was not derived from the axons but was tissue fluid which had accumulated because of a partial obstruction of the vasa nervorum. 


\section{TECHNIQUE OF NERVE CONSTRICTION AND ITS EFFECTS ON CONDUCTION}

The experiments were carried out on seventy-two adult rats. The compression lesion was usually produced on the right sciatic nerve, the left side being used as a control, but in one group of twelve rats lesions were produced on both sides. Under ether anaesthesia the sciatic nerve was exposed in the thigh and a piece of polythene tubing (Sterivac Number 2) eight millimetres long, which had been slit longitudinally, was applied over it. This tube was held in place by slipping over it two threemillimetre pieces of wider (Number 3) tubing which had also been cut longitudinally; the internal diameter of the larger (Number 3) tubing was the same as the external diameter of the smaller (Number 2) tubing (Fig. 1). Although the cross-sectional area enclosed by the intact smaller tube was only about half the cross-sectional area of the average sciatic nerve of a rat, once the tubing had been slit the whole apparatus fitted snugly round the sciatic nerve (Fig. 2) and, as the subsequent experiments show, did not constrict it severely. Although this device did not produce a constant pressure it caused similar changes of only moderately varying intensity in every experiment. In nonc of the experiments was any reaction other than a few adhesions evoked from the surrounding tissue by the polythene.

The motor conductivity of the constricted nerves was studied in twelve rats two to five weeks after the application of the polythene tubing. Under urethane anaesthesia both sciatic nerves of each rat were exposed; a stimulating electrode was applied to the right nerve proximal to the constriction device and another electrode was applied at the same level to the normal left nerve. Oscillographic records from the
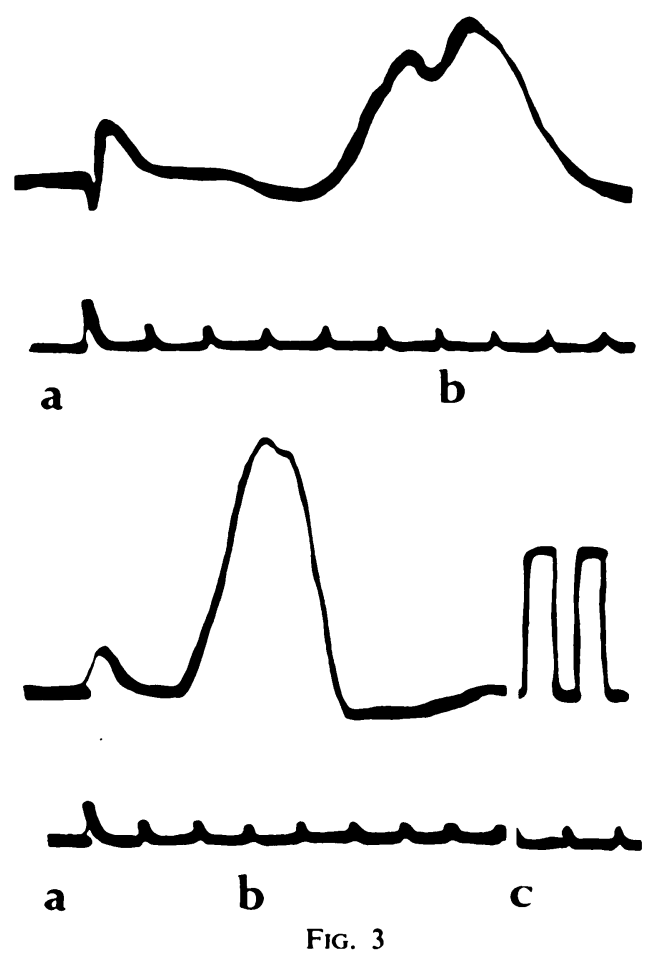

Electromyographic records from both sciatic nerves of rat 98, whose right sciatic nerve had been compressed for four and a half weeks. In each trace the stimulus artefact (a) is followed by the motor unit response (b) after an interval whose duration can be measured from the time base (intervals of 2 milliseconds). The upper trace is from the compressed right sciatic nerve. Note the delayed response from the compressed nerve. c represents a calibration signal of 300 microvolts. muscles of the feet showed a delay in conduction on the constricted side usually amounting to two to four milliseconds (Fig. 3). In four rats repetitive firing occurred after a single minimal stimulus on the constricted side. Identical electromyographic changes occur in carpal tunnel and related syndromes (Simpson 1956). This close resemblance suggests that the structural changes in the experimentally compressed nerves might also be similar to those occurring in the nerve compression syndromes of human pathology.

\section{MACROSCOPIC CHANGES}

All the rats, except for twelve to be referred to later, were killed at intervals of two to five weeks after the initial operation. The nerves which had been constricted for this period had all become swollen proximal and distal to the polythene tube and the swollen portions were moderately hyperaemic. The size of the swollen parts of the nerve varied a little and the amount of swelling was probably related to the efficacy of the constricting device; when it fitted closely round the nerve and had firm, sharp edges, large swellings developed, whereas when the tube fitted loosely, or when its edges were weakened during application, more diffuse swellings were formed.

vol. 46 B, NO. 2, MAY 1964 
Twelve rats were killed within six hours of applying the constriction device. Their sciatic nerves had not become swollen or hyperaemic, although on a few nerves small grooves had formed where the edges of the polythene tubing had rested (Fig. 4). This proves that the swelling was not caused solely by mechanical displacement of axonal material, as might be suggested from the results of Causey's (1948) experiments.

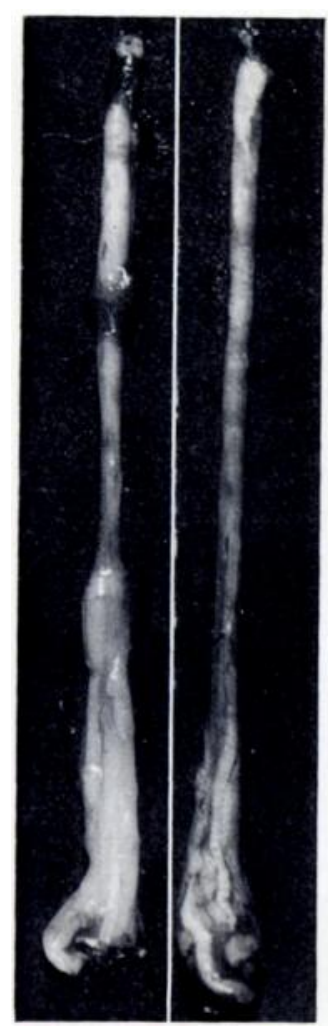

FIG. 4

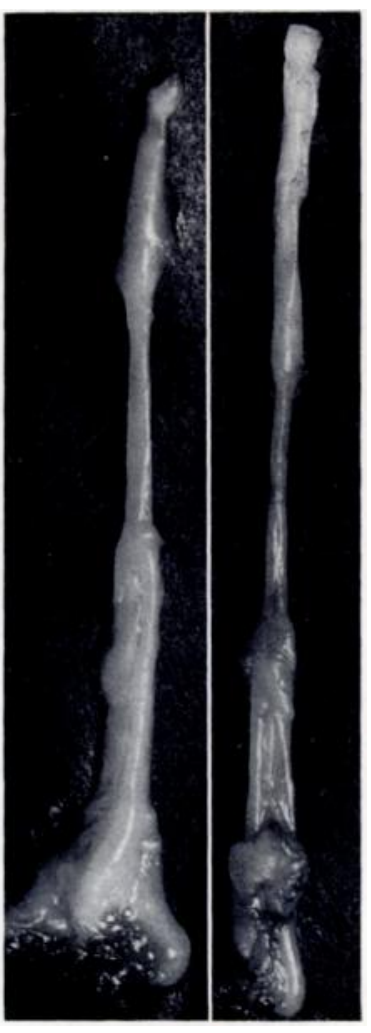

FiG. 5

Figure 4-The sciatic nerves from rat 151 ; the nerve on the left had been compressed for two weeks; that on the right for six hours. The nerve subjected to a long period of compression shows the typical swellings; the other shows no obvious changes. Figure 5-The sciatic nerves of rat 149; both nerves were compressed for three weeks but the shorter nerve was also severed proximal to the compression device. Both nerves show similar swellings proximal and distal to the constriction. The proximal ends of the nerves are directed towards the upper edge of the illustration.

To elucidate matters further, modified experiments were carried out in twelve rats. In this group compression devices were applied to both sciatic nerves and at the same time the nerve on the right side was divided some distance proximal to the polythene tube. After two to five weeks the divided nerve had developed swellings proximal and distal to the compression device similar to those on the opposite nerve (Fig. 5). This shows that the swelling of the nerves is not dependent on the continuity of the axon and is not caused by an obstruction of a flow of axoplasmal fluid.

\section{HISTOLOGICAL CHANGES}

To determine which part of the nerve was involved in the swelling, the compressed right sciatic nerves and the normal left nerves from twelve rats were subjected to detailed microscopic examination. Immediately after each rat had been killed both sciatic nerves were exposed and tied to glass brackets to prevent shrinking; the dissection and excision of the specimens was then completed. Each pair of nerves was fixed, embedded parallel to one another, sectioned transversely and stained as directed by Causey (1948). Sections were taken from each pair of nerves at two-millimetre intervals; the areas of the sections were measured and the number and size of the axons in each section determined.

The sections to be measured were photographed and printed, using an overall magnification of 230 diameters; the photographs were then overprinted with a scale at the same magnification. Using this scale, the print was divided into squares, the unmagnified sides of which were one-tenth of a millimetre. The area of the transverse section was the sum of the squares covered by the nerve.

The axons were too numerous to be counted over the whole area of the section and they were therefore counted on only twelve selected squares, which amounted to about a quarter of the whole area. The squares for counting had to be selected carefully because in the peripheral parts the axons were smaller and more numerous than in the middle of the nerve. To ensure good sampling the area of each section was divided into three equal parts by two concentric circles and within each third those four squares were selected which lay on the two diagonals 
of the photograph (Fig. 6). From this large sample the number of axons in the section and the density of fibres per square millimetre were calculated. As the nerve fibres were being counted they were also graded according to size, using a plastic ruler with holes drilled in it corresponding to diameters of $7,10,14$ and 17 microns; for the purpose of this measurement the myelin sheath was regarded as being part of the nerve fibre.

The findings from the twelve histological experiments are set out in Figure 7. The uppermost group of graphs contrasts the areas of the transverse sections of the normal and compressed nerves; the base line represents the average area of the transverse section of the normal nerves and the graphs show the amount by which the areas of the compressed nerves differed from this average. The middle group of graphs sets out in a similar way the fibre densities; the

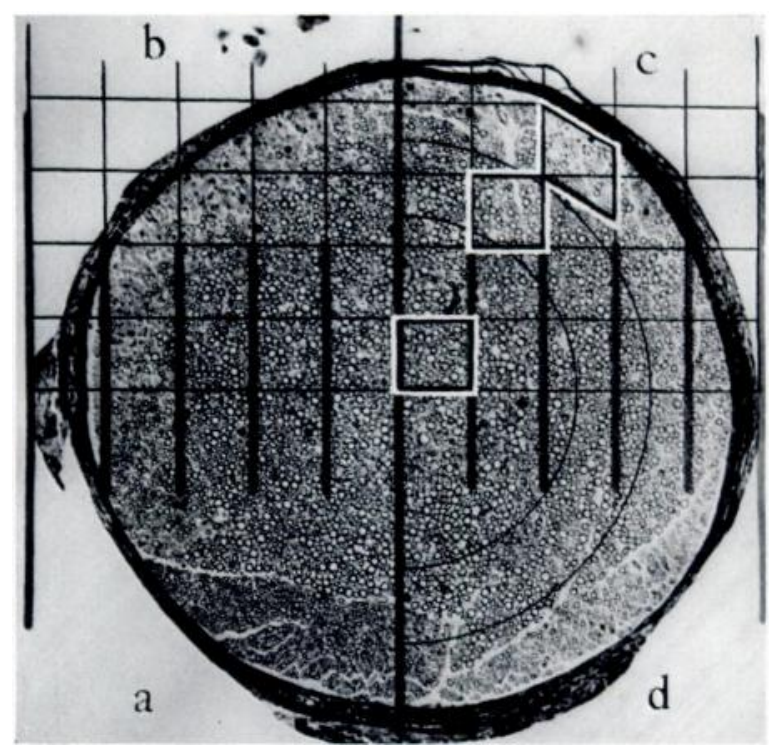

Fig. 6

Transverse section of a nerve illustrating the method of selecting the squares for counting. Quadrant a shows the axons and the overprinted scale at intervals of one-tenth of a millimetre; in quadrants $b$ and $c$ the scale has been extended to mark out squares; in quadrants $c$ and $d$ the circles dividing the area of the nerve into three equal parts have been drawn. Quadrant c shows the three areas selected for counting marked out, one from each third of the nerve and lying close to the diagonal of the photograph.

base line represents the average fibre density in the normal nerves, whilst the graphs represent the fibre densities in the compressed nerves. The lowest graph shows the proportions of fibres of seven microns diameter or smaller (solid line), and fourteen microns diameter and larger (dotted line) in the compressed nerves only.

The uppermost graph (Fig. 7) shows that the polythene tube produced only a slight reduction in the area of the transverse section of the nerve. Relatively little constriction was required to evoke swelling of the nerve proximal and distal to the tube. The comparison of the graphs shows that in the narrowed part of the nerve there were more axons per unit area (middle graph), and that smaller axons predominated (lowest graph). The sections immediately proximal and distal to the constriction device also exhibited this preponderance of smaller fibres, although the area of the section was larger than that of the normal nerve. Further proximally and distally, in the region of greatest swelling, the census of axons followed another 
pattern: the number of axons per unit area was reduced and the proportion of the larger fibres was increased (Figs. 8 to 11 ).

This confirms the findings of the earlier workers that in the constricted parts of the nerve the axons were narrowed and packed more closely, whereas in the swollen parts larger

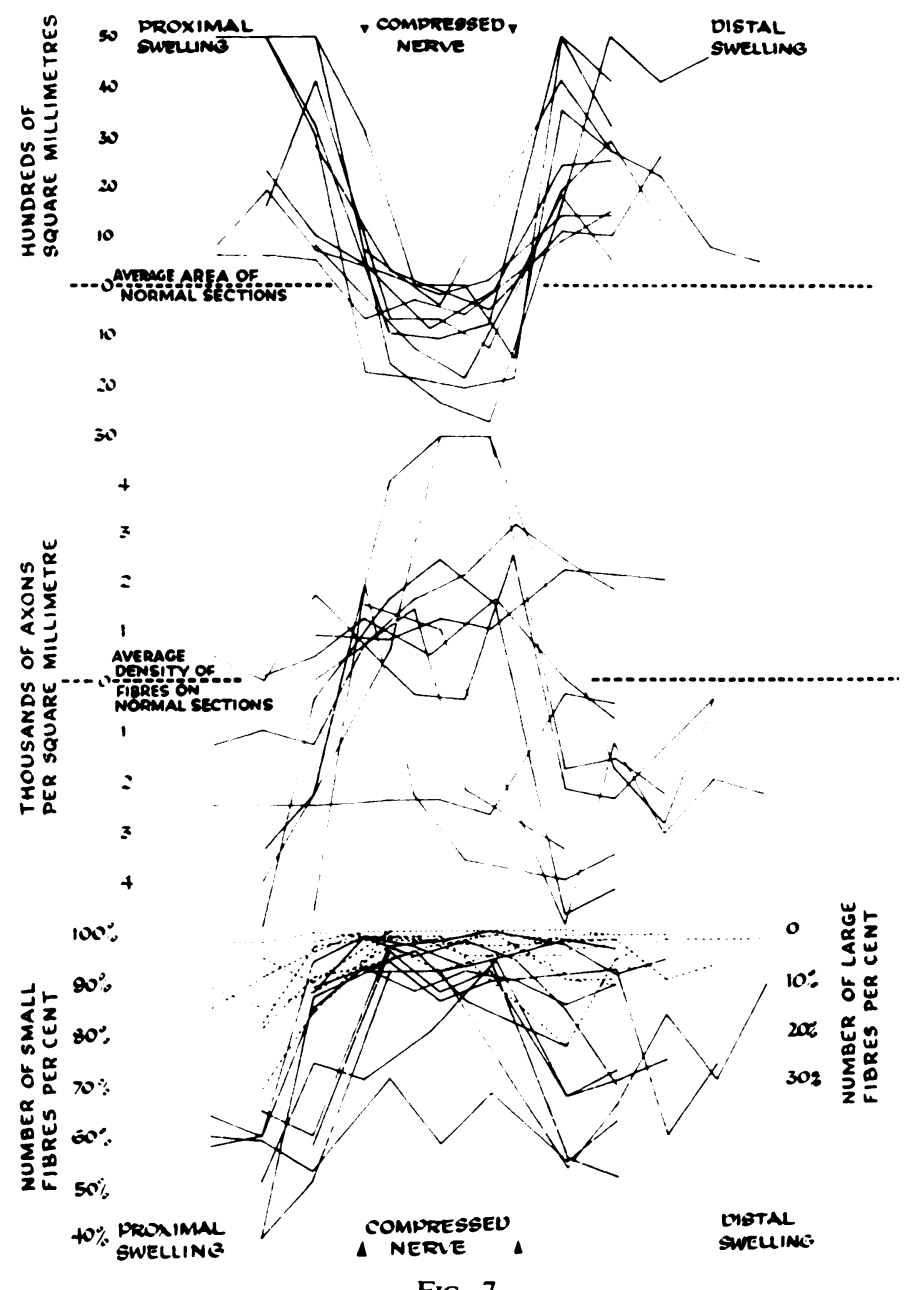

Fig. 7

The results of the histological experiments. The uppermost group of graphs compares the cross-sectional area of the normal and compressed nerves: the base-line represents the average area of the normal nerves, whilst the graphs show the areas of the compressed nerves. The middle group of graphs contrasts the fibre densities of the normal and compressed nerves: the baseline represents the average fibre density of the normal nerves and the graphs show the fibre density of the compressed nerves. The lowest group of graphs shows the proportion of small and large fibres in the compressed nerves; the small fibres were those of 7 microns diameter and less, and are represented by the solid lines, whilst the large axons were those of 14 microns and larger, which are represented by the dotted lines.

axons were separated by increased space which was occupied by " axonal " fluid. To determine the relative contributions to the swelling by the increase in the size of the axons and by the increased amount of fluid between them, the total of the areas of the transverse sections of the axons was calculated and expressed as a proportion of the area of the transverse section 
of the nerve. The areas of the normal sections were approximately equally divided between the axons and the interaxonal space occupied by fluid, whilst in a large majority of sections from the compressed nerves (seventy-one out of ninety-four sections) only a quarter or less

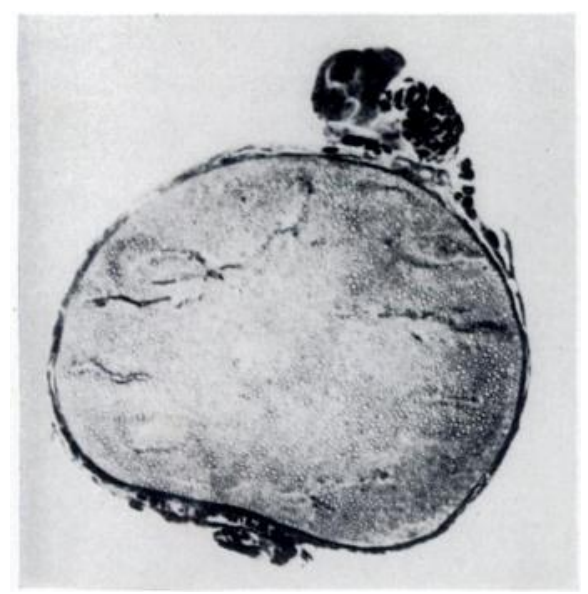

FIG. 8
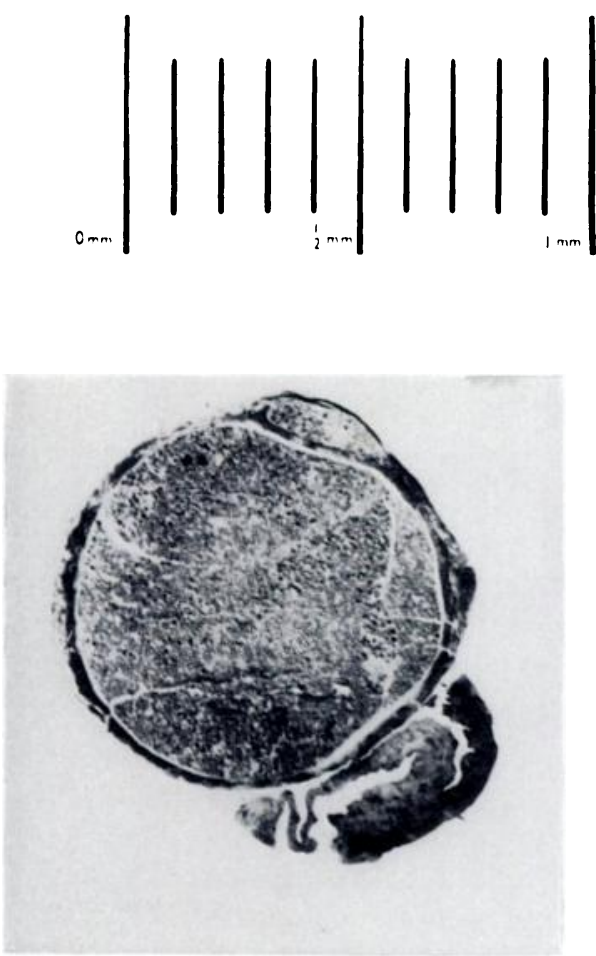

FIG. 10

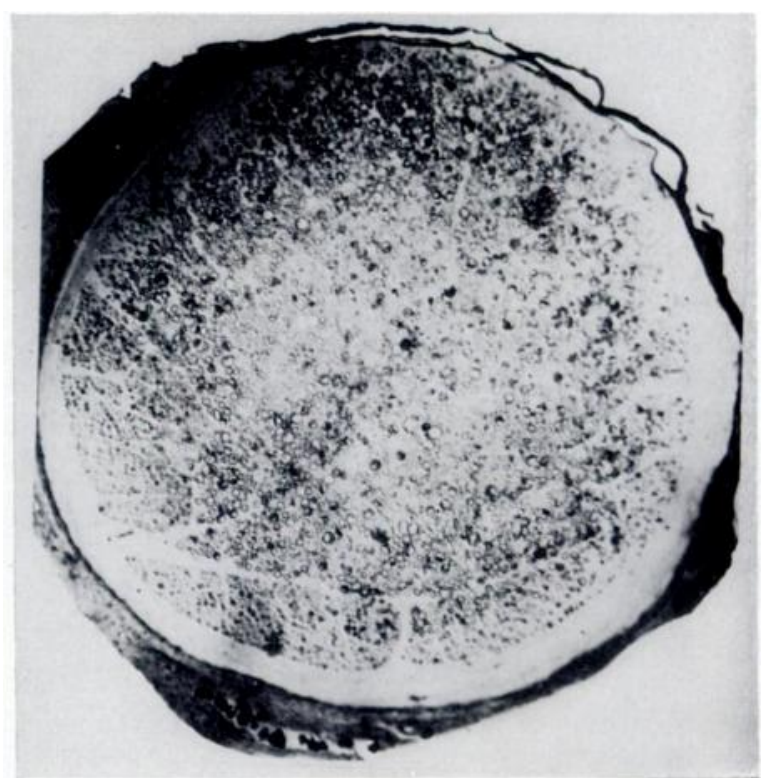

FIG. 9

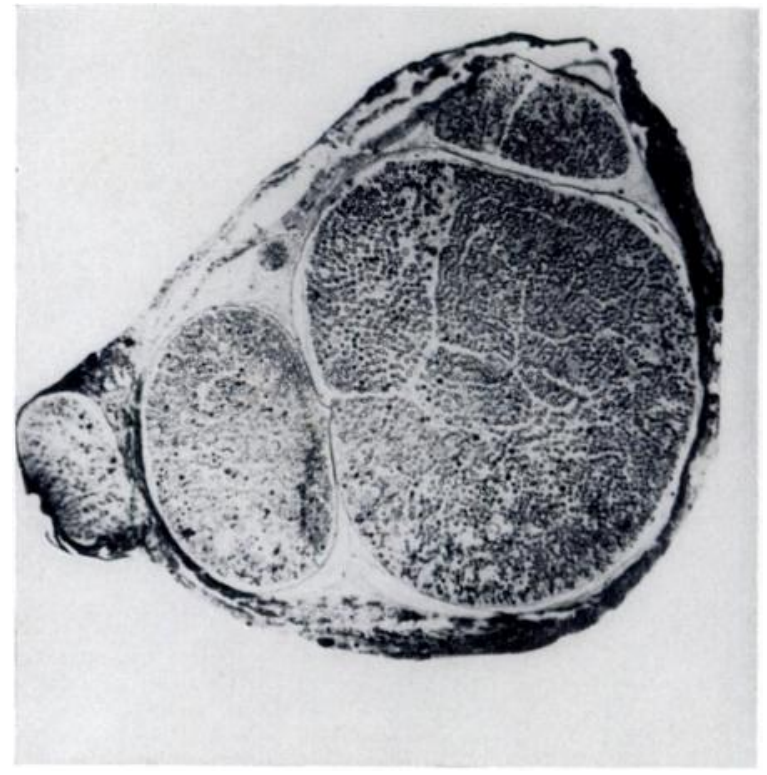

Fig. 11

Microphotographs of transverse sections of the sciatic nerves from rat 67 after three weeks' compression of one nerve. Figure 8-Section through the normal nerve. Figure 9-Section through proximal swelling. Figure 10Section through compressed part of nerve. Figure 11-Section through distal swelling.

of the area was occupied by axons, and three-quarters or more of the section were taken up by the fluid. This makes it abundantly clear that the larger part of the nerve swelling was made up of fluid collected between the axons and that the increased diameter of the axons made only a minor contribution.

VOL. 46 B, NO. 2, MAY 1964 
This change in the proportion of the area taken up by nerve fibres and fluid occurred in sections not only from the swollen parts of the nerve but also from the constricted portions; thus the amount of interaxonal fluid was increased in all parts of the experimental lesion. This fluid collected gradually over a period of time, as was shown earlier in this investigation, when the nerves did not become swollen within six hours of the application of the constriction device. Once this fluid had collected it transmitted the pressure of the constriction device to the axons which became narrowed. It was also noted earlier that the axons were narrowed in sections taken from the swollen parts of the nerve immediately adjacent to the compression device; this is probably caused by the increased amount of fluid, which being confined within the fibrous nerve sheaths, propagated the pressure of the polythene tube a short distance beyond its proximal and distal ends.

This narrowing of the axons can only be regarded as a mechanical effect of the pressure of the surrounding fluid. The axonal substance thus displaced from the compressed parts is bound to collect on either side of the zone of increased pressure producing some increase in the diameter of the axons, as Causey (1948) had already shown. It could not be ascertained whether the displacement of axonal substance accounted for the entire increase in the diameter of the axons.

\section{CHANGES IN THE VASA NERVORUM}

The fact that the swellings developed on all constricted nerves-including those nerves which had been divided proximal to the constriction-made it highly unlikely that the additional fluid was derived from the axons. The only other possible source of fluid was the blood vessels. These were studied in detail by injecting the rats' aortae with a micro-suspension of barium sulphate and then displaying the injected vessels by microradiography of the nerve (Trueta and Harrison 1953).

The vessels of normal nerves and of nerves which had been constricted for from two to five weeks were compared in twenty-four experiments. The vasa in the swollen portions of the compressed nerves were always more numerous and more tortuous than on the normal side, but the vascularity of the constricted part was variable though usually only a little less than on the normal side. Similar changes were observed in the twelve nerves which had been divided proximal to the polythene tube at the same time as the constriction device was being applied (Figs. 12 and 13).

The vasa supplying the part of the sciatic nerve which was compressed in these experiments were derived from the gluteal vessels and the popliteal artery. These vasa nervorum as well as the nerve were compressed by the polythene tube and resistance to blood flow within them must have been increased. In order to maintain the blood flow through the compressed part the pressure in the vessels on either side of the constriction device must have been raised. The greater pressure led to dilation of collateral vessels and caused more fluid to filter through the capillary walls. Thus the fluid between the axons is tissue fluid and the main part of the swelling of the nerve is an oedema; possibly, some of the increase in size of the axons may also be caused by this oedema. This theory, that the swelling of the nerves is caused by partial vascular obstruction and oedema, accounts for the development of swellings distal to, as well as proximal to, a constriction on a nerve and it explains the phenomenon observed in the present experiments that compressed nerves become swollen even though they have been divided proximal to the site of the compression.

\section{CONCLUSIONS}

In this investigation experimental compression lesions have been produced on sciatic nerves of rats. Since the changes in the appearance of these nerves and in their conductivity resemble the changes which occur in nerves affected by carpal tunnel syndrome and ulnar 
neuritis, it may well be that the pathological process in man is similar to that observed in the present study.

The experiments prove that the first effect of gentle nerve compression is partial obstruction of the vasa nervorum leading to hyperaemia and oedema. If these are the initial changes it is hardly surprising that the symptoms of carpal tunnel compression are relieved by elevation, local injection of hydrocortisone and systemic administration of diuretics. As the oedema

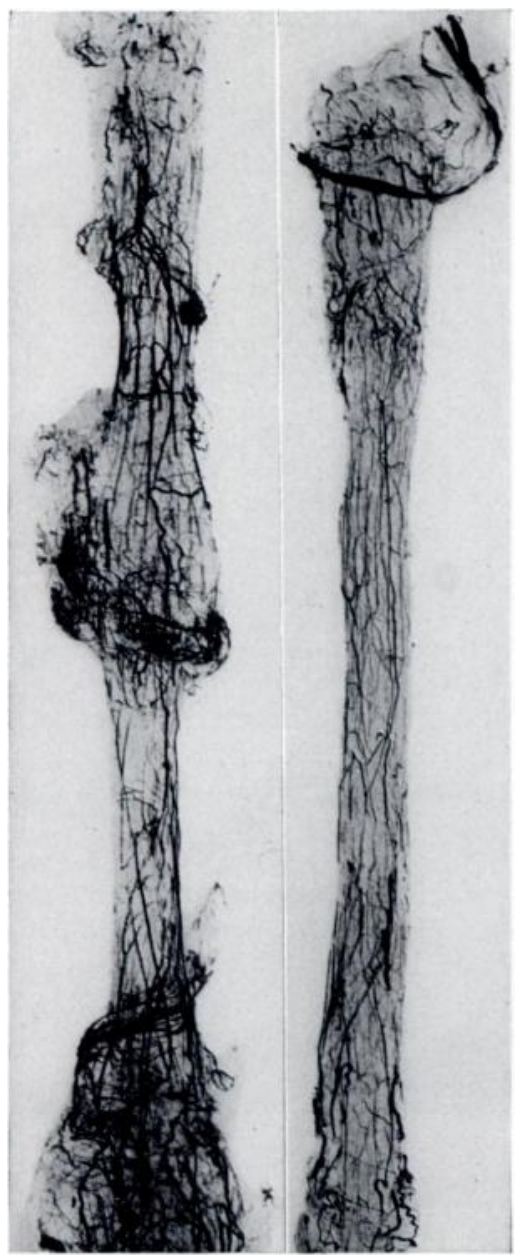

Fig. 12

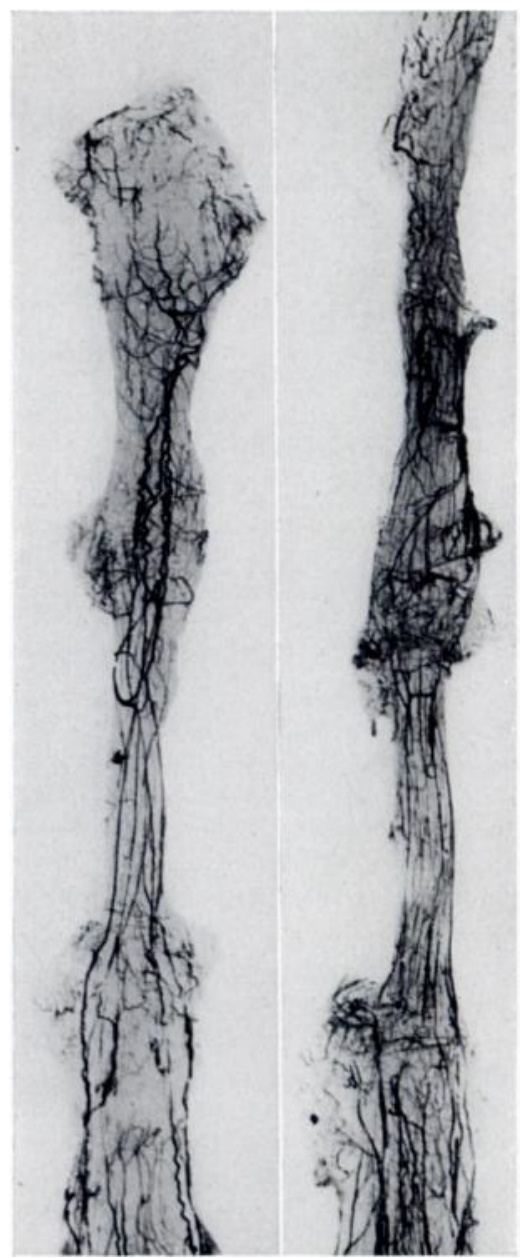

FIG. 13

Microradiographs of nerves in which the arteries have been injected with a microsuspension of barium sulphate. The proximal ends of the nerves are uppermost. Figure 12-Rat 117. Nerve on left was compressed for three weeks; that on right is the normal nerve. Figure 13-Rat 119. Both nerves were compressed for three and a half weeks, but nerve on left was also divided proximal to the constriction when the compression device was applied.

fluid accumulates, the pressure on the axons is increased, narrowing them; this narrowing has been shown to be due to displacement of axonal substance (Causey 1948). The displaced axonal material and also the oedema fluid simply collect proximal and distal to the constriction forming the typical swellings observed by the earlier experimenters (Weiss 1943, 1944, Weiss and Davis 1943, Weiss and Hiscoe 1948, Denny-Brown and Brenner 1944). The present research does not go beyond this point, but it is not difficult to believe with Dustin (1917) that, as happens to other protein exudates, the oedema fluid is invaded by fibroblasts producing 
the fibrous type of lesion originally described by Marie and Foix (1913). Once such fibrosis has occurred it is generally irreversible; it is probably because of this that in long-standing ulnar neuritis decompression or transposition fails to relieve the symptoms completely. If it is accepted that nerve constriction produces oedema followed by fibrosis, then prompt surgical relief of such compression becomes mandatory.

\section{SUMMARY}

1. A method of constricting sciatic nerves of rats was devised which produced lesions resembling macroscopically and electromyographically those of carpal tunnel and related syndromes.

2. The nerves became swollen and hyperaemic proximal and distal to the constriction. The swellings were largely caused by an accumulation of fluid between the axons, but the axons themselves were also increased in size.

3. This accumulation of fluid was an oedema secondary to a partial obstruction of the vasa nervorum.

We should like to acknowledge our great debt to Mr Dillwyn Evans and Professor F. W. Landgrebe for their unfailing help and encouragement. We are grateful to Professor R. G. Harrison and Liverpool University, Dr D. M. D. Evans and the Cardiff Hospital Management Committee, Dr K. N. Lloyd and the Governors of Cardiff Royal Infirmary, Professor H. K. Lloyd, Mr R. D. Garwood and the Department of Metallurgy of the University College of South Wales and Monmouthshire for the use of research facilities. We should like to thank Messrs R. Allison, R. J. Hillard, D. Jones and I. Messenger for technical assistance, Mr J. G. Haddock for photography, and Mrs M. D. V. Hart for secretarial assistance. The Welsh Hospital Board provided the funds for the research.

\section{REFERENCES}

Causey, G. (1948): The Effect of Pressure on Nerve Fibres. Journal of Anatomy, 82, 262.

Denny-Brown, D., and Brenner, C. (1944): Lesion in Peripheral Nerve Resulting from Compression by Spring Clip. Archives of Neurology and Psychiatry, 52, 1.

Dustin, A. P. (1917): Les lésions post-traumatiques des nerfs. Ambulance de "L'Océan," 1, fasc. 2, 71.

MARIE, P., and FoIx, C. (1913): Atrophie isolée de l'éminence thénar d'origine névritique. Rôle du ligament annulaire antérieur du carpe dans la pathogénie de la lésion. Revue Neurologique (Paris), 26, 647.

Simpson, J. A. (1956): Electrical Signs in the Diagnosis of Carpal Tunnel and Related Syndromes. Journal of Neurology, Neurosurgery and Psychiatry, N.S. 19, 275.

Trueta, J., and Harrison, M. H. M. (1953): The Normal Vascular Anatomy of the Femoral Head in Adult Man. Journal of Bone and Joint Surgery, 35-B, 442.

Weiss, P. (1943): Endoneurial Edema in Constricted Nerve. Anatomical Record, 86, 491.

Weiss, P. (1944): Damming of Axoplasm in Constricted Nerve; a Sign of Peripheral Growth in Nerve Fibres. Anatomical Record, 88, 464.

Weiss, P., and Davis, H. (1943): Pressure Block in Nerves Provided with Arterial Sleeves. Journal of Neurophysiology, 6, 269.

Weiss, P., and Hiscoe, H. B. (1948): Experiments on the Mechanism of Nerve Growth. Journal of Experimental Zoolog.', 107, 315. 